\title{
Nonequilibrium calorimetry
}

\author{
Christian Maes $^{1}$ and Karel Netočný ${ }^{2, *}$ \\ ${ }^{1}$ Instituut voor Theoretische Fysica, KU Leuven, Belgium \\ ${ }^{2}$ Institute of Physics, Czech Academy of Sciences, Prague, Czech Republic
}

We consider stationary driven systems in contact with a thermal equilibrium bath. There is a constant (Joule) heat dissipated from the steady system to the environment as long as all parameters are unchanged. As a natural generalization from equilibrium thermodynamics, the nonequilibrium heat capacity measures the excess in that dissipated heat when the temperature of the thermal bath is changed. To improve experimental accessibility we show how the heat capacity can also be obtained from the response of the instantaneous heat flux to small periodic temperature variations.

\section{CALORIMETRY OUT OF EQUILIBRIUM}

Standard thermodynamics deals with equilibrium systems and their energy exchange with the environment as external parameters like the volume or the temperature are changed. If these changes are slow (quasistatic and along equilibria) then the entire scheme simplifies as described by the thermodynamic laws for reversible processes. There heat and entropy get proportional (Clausius heat theorem). As the ability of a specific system to exchange heat is usually given in terms of the heat capacity (the heat contribution from temperature changes) and the latent heat (when temperature is unchanged), heat capacities and latent heats yield important information about the internal structure of the equilibrium system and calorimetry has provided crucial information about microscopic laws and structures.

Concerning possible generalizations, the first (and by now more standard) option is to go beyond quasistatic processes and towards a time-resolved thermodynamics. That can be done within the framework of linear-response and it naturally leads to a frequency-

\footnotetext{
*Electronic address: netocny@fzu.cz
} 
dependent generalization of the heat capacity and related thermodynamic quantities. That was mostly studied for equilibrium systems, with the zero-frequency limit recovering the usual reversible thermodynamic picture. See e.g. [1] and also the very recent [2] in the context of stochastic thermodynamics.

In the present paper we suggest a similar step forward in the study of thermodynamic processes connecting steady states of nonequilibrium systems. In that case the heat exchange contains an inherently irreversible component due to the omnipresent dissipation. One may still apply linear response but fundamentally new features are predicted to appear already in the static (zero-frequency) limit which again corresponds to the quasistatic regime (but now along nonequilibria).

It is important to realize that in driven nonequilibrium systems the heat exchange runs upon a dissipation background. It means that the total heat exchanged with the environment goes virtually to infinity when making the process slower and slower because of its DCcomponent coming from the steady dissipation. Hence, we are really interested not in the (eventually diverging) total heat but in its excess part coming from changes in the temperature and/or other parameters.

On the theoretical side, a natural question arises whether such an excess heat is well defined in the quasistatic regime, in the sense of being essentially insensitive of the actual speed of the process as long as it is slow enough. This question has been answered in the affirmative; see [3, 4]. As a consequence, it is plausible to aim to build up a quasistatic thermodynamic framework based on this excess heat. First of all, it allows to consistently construct a generalization of the heat capacity to nonequilibrium steady states as a static limit of a nonequilibrium frequency-dependent heat capacity; for reversible processes boiling down to the usual concept. We have checked via examples that such a steady heat capacity exhibits some new features when far from thermal equilibrium, for example, it can take negative values. Nevertheless, some more systematic understanding of how these properties reflect the structure of nonequilibrium steady states is still lacking.

Towards an experimental realization, we see various basic ways how to possibly access the quasistatic excess heat and the related steady heat capacity (and similarly for the analogously constructed steady latent heat). First, one may want to measure the excess 
heat directly along a relaxation process to the new steady condition after making a small sudden change of temperature (or other parameters). One then needs to extract the transient part of the dissipated heat, i.e., the one obtained after subtracting the steady "background" dissipation. As a possible variation, instead of measuring the heat directly, it can also be accessed indirectly from measuring the (excess) work done by the driving forces. Yet, main issues to be solved here include the finding of the experimentally most feasible systems on which the temperature can be manipulated on time scales comparable with those of the system itself. The present paper seeks an alternative route however, that is to extend the frequency-dependent calorimetry to truly nonequilibrium systems and to extract the quasistatic excess from its low-frequency behavior. That is the main purpose of the present paper.

We start in the next Section with the theory about the definition of (nonequilibrium) heat capacity. We also include in Section IIC some relevant formulae how to rewrite that for processes modeled as Markov dynamics, in terms of dissipated power. The main result of the paper is in Section III which describes the method of measuring heat capacity via temperature modulation and for which we believe the problem of excess (as a difference between very large quantities) may be avoided. Instead of making the difference of timeextensive heats, we consider there the heat flux as function of time.

\section{NONEQUILIBRIUM THEORY}

We refer to [3, 4] for the initial theory and basic examples of nonequilibrium heat capacities. The basic idea builds on concepts from steady state thermodynamics as in [5, 6]. The result of the present paper is to see in Section III that the specific heat of a system under steady dissipative conditions can be measured by following the dissipated power as a function of time. We start however next with the basic formulæ which rigorously connect the nonequilibrium heat capacity with the excess heat. 


\section{A. Quasistatic excess heat}

Consider a generic thermodynamic system on which external forces perform some work $W$ and which exchanges heat $Q$ with a heat bath at a temperature $T$, so that $W+Q=\Delta U$ is the energy balance. If the system is maintained under fixed nonequilibrium conditions before time zero, then the heat enters at a constant rate $q^{(T)}$ so that $Q_{[-t, 0]}=q^{(T)} t$ when measuring from time $-t<0$, and $W_{[-t, 0]}=-Q_{[-t, 0]}>0$. Both heat and work are time-extensive.

Assume now that we make a measurement of the heat under slow temperature changes starting from time zero. A general quasistatic process can be decomposed in many elementary processes, each one consisting of a tiny sudden warming up (or cooling down) and then followed by a relaxation to new steady conditions. We could sum all the elementary contributions but clearly, for both theoretical and experimental purposes, it is enough to concentrate on one such an elementary process.

Before the sudden change of temperature from $T$ to $T+\mathrm{d} T$ at time zero, the system was in the steady state at the temperature $T$. After the change, it undertakes a relaxation to the new steady state at $T+\mathrm{d} T$. That is a transient process and the heat $Q_{[0, t]}$ is no longer purely extensive but it contains a transient part as well. The latter can be extracted by comparing with the steady heat under the new stationary conditions, which is $q^{(T+\mathrm{d} T)} t$. That transient contribution along the complete relaxation process,

$$
\delta Q^{\mathrm{ex}}=\lim _{t \rightarrow \infty}\left(Q_{[0, t]}-q^{(T+\mathrm{d} T)} t\right)
$$

is called an excess heat. Note that we really have to subtract the steady heat as corresponding to the new temperature $T+\mathrm{d} T$ since the dissipation rate can be (and typically is) temperature-dependent. Under equilibrium conditions the latter would be just zero and the excess heat coincides with the total heat exchange along the elementary process. In contrast, out of equilibrium we take the difference of large (in the limit, infinite) quantities. In practice, one surely performs no time limit but, instead, let the relaxation run till it is "essentially finished". If $\tau$ is a characteristic time of relaxation then the excess heat $\delta Q^{\mathrm{ex}}$ is to be compared with the steady heat $q^{(T)} \tau$. Obviously, if $q^{(T)} \tau \gg\left|\delta Q^{\mathrm{ex}}\right|$ then one can hardly expect the excess heat to be distinguishable against the steady dissipation background. 


\section{B. Steady heat capacity}

The steady heat capacity quantifies the extra heat needed for the system to accommodate to a unit temperature change,

$$
C(T)=\frac{\delta Q^{\mathrm{ex}}}{\mathrm{d} T}
$$

Analogously, one can consider more general quasistatic processes including also the change of other thermodynamic parameters, which would then lead to a nonequilibrium generalization of the latent heat (capacities). All these quantities naturally supplement the incoming heat flux $q^{(T)}$ and provide a more complete characterization of the nonequilibrium steady state and its thermal sensitivity to external perturbations.

Although heat is a primary quantity here, we can as well consider the excess work defined analogously as

$$
\delta W^{\mathrm{ex}}=\lim _{t \rightarrow \infty}\left(W_{[0, t]}-w^{(T+\mathrm{d} T)} t\right)
$$

where always $W_{[0, t]}+Q_{[0, t]}=U(t)-U(0)$. Since the steady power on the system is just $w^{(T+\mathrm{d} T)}=-q^{(T+\mathrm{d} T)}$, we can relate (3) with (1) in the balance $\delta W^{\mathrm{ex}}+\delta Q^{\mathrm{ex}}=\mathrm{d} U$. Hence, the steady heat capacity (2) can also be written in the form

$$
C(T)=\frac{\partial U}{\partial T}-\frac{\delta W^{\mathrm{ex}}}{\mathrm{d} T}
$$

where the first term is a usual temperature-energy response. Under equilibrium conditions such as constant volume and/or other thermodynamic coordinates, the second term vanishes and the familiar equilibrium formula is recovered.

\section{For Markov processes}

Note that for mesoscopic systems the work and heat are fluctuating quantities and hence in the above analysis, e.g. $W_{[0, t]}$ in $(3)$ is to be understood as a statistical average. To be clear about that mesoscopic theory and depending on the theoretical model, we add here a formulation of the above for Markov jump processes.

We imagine a system with a few degrees of freedom, like multilevel systems, where the state $x$ of the system is uniquely associated to an energy level $E(x)$. In addition, work 
$F(x, y)$ is done by the system during each transition $x \rightarrow y$. Finally, the system is in a thermal environment at a temperature $T$. As model we consider a Markov jump process with transition rates

$$
\begin{aligned}
& k^{T}(x, y):=a_{T}(x, y) e^{\frac{\beta}{2}[E(x)-E(y)+F(x, y)]}, \quad \beta:=\frac{1}{k_{B} T} \\
& F(x, y)=-F(y, x), \quad a_{T}(x, y)=a_{T}(y, x)
\end{aligned}
$$

between states $x \rightarrow y$. We are careful to indicate the dependence on the temperature $T$ of the external thermal bath. The energy function $E$ is related to the reversible part of the transition, while $F$ refers to the irreversible part. The expected instantaneous dissipated power when in state $x$ equals

$$
\mathcal{P}^{T}(x)=\sum_{y} k^{T}(x, y) F(x, y)
$$

and the dissipated work is in the balance, per trajectory,

$$
\sum_{s} F\left(x_{s^{-}}, x_{s}\right)=Q-E\left(x_{t}\right)+E\left(x_{0}\right)
$$

where the sum is over jump times $s \in[0, t]$ and $Q$ is the heat into the system over times $s \in[0, t]$. All that is consistent with the condition of local detailed balance which requires that the antisymmetric part of $\log k^{T}(x, y) / k^{T}(y, x)$ be given by the entropy flux per $k_{B}$ over the transition $x \rightarrow y$ into the environment at temperature $T$.

The excess heat (1)-(2) is the difference, for $T^{\prime}=T+\mathrm{d} T$,

$$
\langle Q\rangle-\langle Q\rangle_{T^{\prime}}=C(T) \mathrm{d} T
$$

By $\langle Q\rangle$ we mean the entering heat average over all trajectories in $[0, t]$ where we started from temperature $T$ (in the heat bath) and move on at positive times with the heat bath at temperature $T^{\prime}$. The average $\langle Q\rangle_{T^{\prime}}$ is the steady heat at that temperature $T^{\prime}=T+\mathrm{d} T$. Note however that (8) does not appear promising as such for experimental use - heat is fluctuating and the variance may be growing with time. That is also why we will have Section 【II.

Following the reasoning outlined in the previous subsections we arrive at

$$
C(T) \mathrm{d} T=\langle E\rangle_{T^{\prime}}-\langle E\rangle_{T}-\left\langle\int_{0}^{\infty}\left[\mathcal{P}^{T^{\prime}}\left(x_{s}\right)-\left\langle\mathcal{P}^{T^{\prime}}(x)\right\rangle_{T^{\prime}}\right] \mathrm{d} s\right\rangle
$$


where the average $\langle\cdot\rangle$ is over the Markov process starting at temperature $T$ but running at temperature $T^{\prime}$. For a final rewriting we introduce the function

$$
V_{T^{\prime}}(x):=\left\langle\int_{0}^{\infty}\left[\mathcal{P}^{T^{\prime}}\left(x_{t}\right)-\left\langle\mathcal{P}^{T^{\prime}}(x)\right\rangle_{T^{\prime}}\right] \mathrm{d} t \mid x_{0}=x\right\rangle_{T^{\prime}}
$$

where we condition on starting at time zero from $x$ for running the nonequilibrium system dynamics at temperature $T^{\prime}$. Obviously, as we initially draw $x$ from the steady nonequilibrium at temperature $T$,

$$
\left\langle\int_{0}^{\infty}\left[\mathcal{P}^{T^{\prime}}\left(x_{t}\right)-\left\langle\mathcal{P}^{T^{\prime}}(x)\right\rangle_{T^{\prime}}\right] \mathrm{d} t\right\rangle=\left\langle V_{T^{\prime}}\right\rangle_{T}=\sum_{x} V_{T^{\prime}}(x) \rho_{T}(x)
$$

where $\rho_{T}$ denotes the stationary nonequilibrium distribution at temperature $T$. Since by construction

$$
\left\langle V_{T}\right\rangle_{T}=\sum_{x} V_{T}(x) \rho_{T}(x)=0
$$

we conclude that (9) equals

$$
C(T)=\frac{1}{\mathrm{~d} T}\left[\langle E\rangle_{T^{\prime}}-\langle E\rangle_{T}-\sum_{x}\left\{V_{T^{\prime}}(x)-V_{T}(x)\right\} \rho_{T}(x)\right]
$$

or, for short,

$$
C(T)=\frac{\mathrm{d}}{\mathrm{d} T}\langle E\rangle_{T}-\left\langle\frac{\mathrm{d}}{\mathrm{d} T} V_{T}(x)\right\rangle_{T}
$$

That is the main formula for the nonequilibrium heat capacity under constant system parameters (such as the volume) making precise formula (4) for Markov processes. Note again that its first term is the usual equilibrium expression, estimating the change in stationary energy under a temperature change: for $F=0$, the system satisfies detailed balance, which we refer to as equilibrium. The second term concerns the excess in irreversible heat (10) which also changes with temperature for fixed forcing.

The formula (11) can be rigorously derived under quasistatic temperature changes, see [3]. For diffusion processes similar expressions hold, as for example made explicit in eq. 3.5 in [7]. For dissipative mechanical systems we have the usual $\mathcal{P}^{T}(p, q)=F(q) \cdot p$ for nonconservative force $F$ (which can depend implicitly on $T$ ) and states $x=(p, q)$.

$\underline{\text { Remarks }}$

1. If there is a function $\Phi(T)$ depending on the environment's parameters so that the left-hand side of (8) (the excess heat) would be equal to

$$
\langle Q\rangle-\langle Q\rangle_{T^{\prime}}=g(T)\left[\Phi\left(T^{\prime}\right)-\Phi(T)\right]
$$


then,

$$
C(T) \mathrm{d} T=g(T) \mathrm{d} \Phi(T), \quad C(T)=g(T) \frac{\mathrm{d} \Phi}{\mathrm{d} T}
$$

That holds in equilibrium with $g(T)=T, \Phi(T)=S(T)$ the thermodynamic entropy at temperature $T$, as expressed in the Clausius' heat theorem. It also holds in first order around equilibrium (and sometimes even to second order) as follows from steady state thermodynamics, [5, 6].

2. When $V_{T}(x)=v(x)-\varphi(T)$, then

$$
C(T)=\frac{\mathrm{d}}{\mathrm{d} T}\left(\langle E\rangle_{T}+\varphi(T)\right)
$$

The formula 12 holds approximately to the extent that $V_{T}(x)+\varphi(T)$ would be a slowly varying function of $T$, which can be expected in some temperature-ranges; see [4].

3. Close-to-equilibrium expressions for the heat capacity $C(T)$ can be obtained by invoking McLennan ensembles to approximate $\langle\cdot\rangle_{T}$-expectations, see [7]. As it happens, in linear order around equilibrium (first order in the magnitude of $F$ ), the correction to the Gibbs ensemble is exactly given by $V_{T}$ :

$$
\rho_{T}(x) \propto \exp \left\{-\beta\left[E(x)+V_{T}(x)\right]\right\}
$$

Per consequence, close-to-equilibrium, (11) is given by

$$
C(T) \simeq \frac{\partial}{\partial T}\langle E\rangle_{T}-\frac{1}{T}\left(\langle E\rangle_{T}-\langle E\rangle_{T}^{\mathrm{eq}}\right)
$$

up to linear order in the nonequilibrium driving $F$.

\section{TEMPERATURE-HEAT RESPONSE}

The heat can generally be resolved into the time-dependent flux as $Q=\int J_{t}^{Q} \mathrm{~d} t$. Initially we have a heat current $J_{0}^{Q}=q^{(T)}$ into the system (equal to minus the steady rate of dissipation at temperature $T$ ). Let us now modulate the temperature, $T_{s}=T+h_{s}$, at times $s \geq 0$. We work within linear response to state that the heat current at time $t>0$ is

$$
J_{t}^{Q}=J_{0}^{Q}+\lambda_{\infty} h_{t}+\int_{0}^{t} \lambda_{s} h_{t-s} \mathrm{~d} s
$$


The function $\lambda_{t}$ is a temporal temperature-heat "admittance", assumed to decay fast enough in time; $\lambda_{\infty}$ accounts for the immediate, non-delayed response. The temperaturedependence of the steady heat current can be obtained by taking the limit $t \uparrow \infty$ under which $J_{t}^{Q} \rightarrow J_{\infty}^{Q}$ becomes the steady heat per unit time at temperature $T^{\prime}$.

Let us again take the special case where the temperature suddenly changes at time zero from $T$ to $T^{\prime}=T+\mathrm{d} T$ (i.e., $h_{s}=\mathrm{d} T$ for $s>0$ ). From (8) the heat capacity $C(T)$ satisfies

$$
\int_{0}^{\infty}\left(J_{t}^{Q}-J_{\infty}^{Q}\right) \mathrm{d} t=C(T) \mathrm{d} T
$$

so that (14) yields

$$
C(T)=\int_{0}^{\infty} \mathrm{d} t \int_{t}^{\infty} \lambda_{s} \mathrm{~d} s=-\int_{0}^{\infty} t \lambda_{t} \mathrm{~d} t
$$

which expresses the heat capacity in terms of the admittances.

On the other hand, the difference in steady heat currents is

$$
J_{\infty}^{Q}-J_{0}^{Q}=: \sigma \mathrm{d} T
$$

with, again from (14),

$$
\sigma=\lambda_{\infty}+\int_{0}^{\infty} \lambda_{t} \mathrm{~d} t
$$

Of course, a continuous small change of temperatures can be seen as a sequence of temperature changes $\mathrm{d} T_{s}=\dot{h}_{s} \mathrm{~d} s$. To illustrate how the above can be combined to measure $C(T)$, let us take a temperature protocol $h_{s}=T_{s}-T=\epsilon \sin (\omega s)$ with some small unit $\epsilon$ of temperature and frequency $\omega$. Imagine that one measures the time-dependence (with some $\gamma>0)$

$$
J_{t}^{Q}=q^{(T)}+\epsilon\left[\sigma_{1}(\omega) \sin (\omega t)+\sigma_{2}(\omega) \cos (\omega t)\right]+O\left(e^{-\gamma t}\right)
$$

defining the $\sigma_{1,2}(\omega)$ as the in- and out-phase components of the temperature-sensitivity of the dissipation. Comparing with (14), they are related to the admittance $\lambda_{t}$ by the FourierLaplace transform

$$
\sigma_{1}(\omega)+i \sigma_{2}(\omega)=\lambda_{\infty}+\int_{0}^{\infty} e^{-i \omega t} \lambda_{t} \mathrm{~d} t
$$

From (16) we get $\sigma_{1}(\omega=0)=\sigma, \sigma_{2}(\omega=0)=0$, and from 15),

$$
\left.\frac{\partial \sigma_{1}}{\partial \omega}\right|_{\omega=0}=0,\left.\quad \frac{\partial \sigma_{2}}{\partial \omega}\right|_{\omega=0}=C(T)
$$


Hence, combining that with (17), the low-frequency asymptotics of the heat current response is

$$
J_{t}^{Q}=J_{0}^{Q}+\epsilon\left[\sigma \sin (\omega t)-C(T) \omega \cos (\omega t)+O\left(\omega^{2}\right)\right]+O\left(e^{-\gamma t}\right)
$$

We see that the (quasistatic) steady heat capacity provides the leading low-frequency (outphase) correction to the steady (in-phase) linear temperature-heat relation. This also indicates how the steady heat capacity can be detected and measured from the response to slow periodic temperature variations.

Note that this is nothing but a frequency-dependent calorimetry restricted to low frequencies, the only difference being that in the usual equilibrium setup $J_{0}^{Q}=q^{(T)}$ vanishes. In contrast, around a steady nonequilibrium the latter provides the dominant (for $\omega \rightarrow 0$ ) contribution to the heat flux, whereas the heat capacity becomes the next correction.

\section{CONCLUSIONS}

Thermal properties of nonequilibria appear essential in the program of steady state thermodynamics. Calorimetry of nonequilibrium systems may be developed to provide a useful characterization of the change in a material's thermal properties when driven away from equilibrium conditions, [8, 9]. Nonequilibrium heat capacities can be consistently defined in terms of the notion of excess heat, or from how the steady dissipated power varies with temperature. We have seen how temperature modulation for nonequilibria gives access to that information via the time-dependence of the instantaneous heat flux.

[1] J. K. Nielsen and J. C. Dyre, Fluctuation-dissipation theorem for frequency-dependent specific heat. Phys. Rev. B 54, 15754 (1996).

[2] M.J. de Oliveira, Complex heat capacity and entropy production of temperature modulated systems. arXiv:1905.10306v1 [cond-mat.stat-mech]

[3] E. Boksenbojm, C. Maes, K. Netočný, and J. Pešek, Heat capacity in nonequilibrium steady states. Europhys. Lett. 96, 40001 (2011).

[4] J. Pešek, E. Boksenbojm, and K. Netočný, Model study on steady heat capacity in driven stochastic systems. Cent. Eur. J. Phys. 10(3), 692-701 (2012). 
[5] Y. Oono and M. Paniconi, Steady state thermodynamics. Prog. Theor. Phys. Suppl. 130, 29 (1998).

[6] T.S. Komatsu, N. Nakagawa, S.I. Sasa and H. Tasaki, Steady State Thermodynamics for Heat Conduction - Microscopic Derivation. Phys. Rev. Lett., 100, 230602 (2008).

-, J. Stat. Phys. 134, 401 (2009).

[7] C.Maes and K.Netočný, Rigorous meaning of McLennan ensembles. J. Math. Phys. 51, 015219 (2010).

[8] Handbook of Thermal Analysis and Calorimetry: From macromolecules to man, Volume 4, Eds.Patrick Kent Gallagher, Michael E. Brown and Richard B. Kemp, Elsevier, 1999.

[9] The nature of biological systems as revealed by thermal methods, Volume 5 of Hot topics in thermal analysis and calorimetry, Ed. Dénes Lörinczy, Springer, 2004. 